\title{
Mismatch between Sites of Incidence of Out-of-Hospital Cardiac Arrest and Locations of Installed Automated External Defibrillator in the Tokyo Metropolitan Area
}

\author{
Kyoko Tsukigase ${ }^{1}$, Hideharu Tanaka, ${ }^{1,2}$ Hiroshi Takyu² \\ ${ }^{1}$ Research Institute of Disaster Management and Emergency Medical System, Kokushikan University, Tokyo, Japan \\ ${ }^{2}$ EMS System, Graduate School of Kokushikan University, Tokyo, Japan \\ Email: k-tsukigase@kokushikan.ac.jp
}

How to cite this paper: Tsukigase, K., Tanaka, H. and Takyu, H. (2017) Mismatch between Sites of Incidence of Out-of-Hospital Cardiac Arrest and Locations of Installed Automated External Defibrillator in the Tokyo Metropolitan Area. World Journal of Cardiovascular Diseases, 7, 185-194. https://doi.org/10.4236/wjcd.2017.76017

Received: April 21, 2017

Accepted: June 12, 2017

Published: June 15, 2017

Copyright $\odot 2017$ by authors and Scientific Research Publishing Inc. This work is licensed under the Creative Commons Attribution International License (CC BY 4.0).

http://creativecommons.org/licenses/by/4.0/

\begin{abstract}
Background: Since 2004, the number of installed Automated External Defibrillator (AED) has been increased in Japan annually, the cumulative number of sold AED more than 600,000 units by 2016. Despite there have been about 130,000 out of hospital cardiac arrest annually, there have only 1302 cases delivered defibrillation by bystanders at the scene. Therefore, we investigate that number of AED installation and usage rate for Out of Hospital Cardiac Arrest (OHCA) patients. Methods: Retrospective metropolitan wide cohort study. Subject: Total 13,364 OHCA patients in the Tokyo Metropolitan area from Jan. 1 through Dec. 31 in 2012 were eligible for theses analyses. Also, OHCA occurrence place and AED usage rate were studied. Results: $82.8 \%$ of OHCA occurred at residences, $10 \%$ at outdoors, $6.4 \%$ at indoors, only $0.7 \%$ at schools. In the other hand, highest bystander's CPR rates were found in sports facilities and schools (71.4\%). The installation rate of AEDs in residences was 9.6\%, instead of highest incidence for OHCA patients, school and sports institute made up $21.4 \%$ of AED installations, but only $1.0 \%$ of the incidences of cardiac arrest. We found that there is a mismatch between incidence sites of cardiac arrest and locations of installed AED. Discussion: It is essential to increase the number of AED installations. Furthermore, it is desirable to install AEDs in locations where cardiac arrest is liable to occur following guidelines for the proper placement of AEDs, and important to further spread BLS education among the general citizens.
\end{abstract}

\section{Keywords}

AED, Cardiac Arrest, Defibrillation, Bystander, First Aid 


\section{Introduction}

The use of Automated External Defibrillator (AED) by non-healthcare professionals has been approved in Japan from July 2004 [1]. The cumulative number of sales for AEDs throughout Japan in 2004 was 1307 units, but ten years later, in 2014, that number had become 516,135 units [2]. Among these, the Tokyo metropolitan area had the highest installations of AEDs in Japan at 40,504 units as of December 2013 [3].

On the other hand, the number of AEDs used in 2012 by bystanders to deliver the shock at the scene of incidence for cardiac arrest patients was 1802 [4]. This is only $4.9 \%$ of the 364,959 installed in Tokyo metropolitan area at the time. It suggested that the Public Access Defibrillators have been not being fully effective utilized.

Thus, we investigated that reason of the low usage rate instead of the high number of installations was perhaps mismatch of the locations of installed AED and the sites of incidence for cardiac arrest patients. No one compared with that before.

\section{Objective}

The incidence for Out of Hospital Cardiac Arrest (OHCA) patients transported by Tokyo Fire Department (TFD) and bystander's CPR rates in the Tokyo metropolitan area were studied. In addition, mismatch between the actual sites of incidence of OHCA and the locations of AED installations.

\section{Method and Subjects}

\subsection{Study Design}

Retrospective metropolitan wide cohort study.

\subsection{Subject}

From January 1 through December 31, 2012, total of 13,364 OHCA transported data adopted from in Tokyo Metropolitan area (covered by the Tokyo Fire Department) were eligible for these analyses.

\subsection{Methods}

Ambulance crews of TFD recorded each site of OHCAs in their report. Sites of OHCA incidence were analysed with five categories as follows, "Residences" which including home, nursing homes, and hotels, "Indoors", "Schools", "Outdoors" and "Others" according to annual reports the Tokyo Fire Department Currently State of Emergencies (2012) published in 2013 [5] (Table 1).

The nine items were corrected and analysed as follows: 1 ) rate of the witnesses cardiac arrest (\%), 2) rate of bystander CPR applied (\%), 3) rate of bystander first aid, 4) rate of defibrillation (including defibrillation by Emergency Medical Service (EMS), 5) rate of defibrillation when witnessed, 6) rate of return of spontaneous circulation(ROSC) prior to arrival at the hospital, 7) rate of return 
Table 1. Categorization of sites of incidence for cardiac arrest.

\begin{tabular}{cl}
\hline Residences & \multicolumn{1}{c}{ Residences, nursing homes, lodgings } \\
\hline Indoors & $\begin{array}{l}\text { Company offices and factories; sales and service facilities; entertainment and } \\
\text { amusement facilities; health, rehabilitation and cosmetic facilities; medical } \\
\text { facilities; art and culture facilities; religious facilities and funeral homes; } \\
\text { government and administrative facilities }\end{array}$ \\
Outdoors & $\begin{array}{l}\text { Roads, vehicles and transportation facilities; natural environments and open } \\
\text { spaces; construction sites }\end{array}$ \\
Schools & Day care facilities and schools; sports facilities; parks and amusement parks \\
Others & Others \\
\hline
\end{tabular}

of spontaneous circulation (ROSC) prior to arrival at the hospital when witnessed, 8) one-month survival rate, and 9) one-month survival rate when witnessed according to the Utstein style, comparison was performed with odds ratio with "Outdoors" as the standard.

Moreover, first aid applied by bystanders was limited effective treatments such as artificial respiration, chest compression or defibrillation with AED for the OHCA patients according to the current definitions in Current State of Emergencies published by the Tokyo Fire Department.

Further, the location of Installed AED searching on the website of the Japan Foundation for Emergency Medicine [6] was used for examining the locations of AEDs installed.

These were grouped into the five categories for the sites of incidence above, the percentages among all registered AEDs were calculated and a comparison was made with the actual sites of incidence for cardiac arrest.

\subsection{Ethical Issue}

This study was approved after a review by the Kokushikan University Ethics Committee. Since the data adopted in this study from the Tokyo Fire Department Current State of Emergencies, that not includes any personal information.

\subsection{Statistical Analysis}

Microsoft ${ }^{\circledR}$ Excel $^{\circledR}$ for Mac 2011 was used for data and processing.

Comparison was performed with odds ratio (95\% confidence interval listed in parentheses) for simple tabulation and statistical analysis.

\section{Results}

\subsection{Patients Background}

Total 13,364 cardiac arrest patients transported within the Tokyo metropolitan area in 2012. As for the backgrounds of the 13,364 patients, the average age was 72.8 years old, for gender was $57.2 \%$ (7649) were men and $42.8 \%$ (5715) were women, the cardiac arrest was witnessed for $45.1 \%$ (6021), bystander's CPR for $27.7 \%$ (3704), defibrillations for $9.9 \%$ (1320), ROSC prior to arrival to the hos- 
pital for 9.2\% (1228) and the one-month survival rate was 3.3\% (442).

\subsection{Sites of Incidence for OHCA}

The incidences of for OHCA occurrence place were compared. The cardiac arrest occurred at "Residences" for 11,068 patients (82.8\%), "Outdoors" for 1334 patients (10.0\%), "Indoors" for 859 patients (6.4\%), "Schools" for 98 patients $(0.7 \%)$ and "Others" for 5 patients $(0.0 \%)$.

The average ages of the OHCA was the highest in the "Residences" at $77.5 \mathrm{yr}$, followed by "Others" at $62.6 \mathrm{yr}$, "Indoors" at $61.6 \mathrm{yr}$, and "Outdoors" at $55.7 \mathrm{yr}$. and lowest in the "Schools" at $49.5 \mathrm{yr}$, respectively (Table 2).

\subsection{Locations of Installed AED in the Tokyo Metropolitan Area}

Total 21,017 AEDs registered in the Tokyo metropolitan area as of June 2013 with five categories of the location of Installed AED into matched by the website information of the Japan Foundation for Emergency Medicine (6). The results showed that "Indoors" had the highest percentage at $62.4 \%$ (13,124 units), followed by "Schools" at 21.4\% (4500), "Residences" at 9.6\% (2012), "Outdoors" at $5.3 \%$ (1119) and "Others" at 1.2\% (262), respectively.

Comparing the incidence of OHCA with percentage for PAD for "Residences" was extremely low at $9.6 \%$ even though this category had the highest percentage of incidences of cardiac arrest at $82.8 \%$. The percentage of AED installations for "Indoors" was very high at $62.4 \%$ even though the rate of incidence for cardiac arrest was $6.0 \%$. "Schools" made up $21.4 \%$ of AED installations, but only $1.0 \%$ of the incidences of cardiac arrest (Figure 1).

\subsection{Comparisons of OHCA Sites and of 9 Utstein Data Items}

Table 3 indicates comparisons of OHCA occurred sites of incidence with 9 Utstein data item.

1) Witnesses OHCA

The witnessed OHCA most commonly at "Schools" at $80.6 \%$, followed by "Indoors" at $66.0 \%$, "Outdoors" at $56.8 \%$ and "Other" at $60.0 \%$. It was witnessed the least at "Residences" at $41.7 \%$. The odds ratio ("Outdoors" as the standard) was highest for "Schools" at 3.16 (1.89 - 5.27).

Table 2. Number and average ages of cardiac arrest patients by site of incidence.

\begin{tabular}{ccccc}
\hline & $\begin{array}{c}\text { Site of } \\
\text { incidence }\end{array}$ & $\begin{array}{c}\text { Total number } \\
\text { (patients) }\end{array}$ & $\begin{array}{c}\text { Percentage } \\
(\%)\end{array}$ & $\begin{array}{c}\text { Average age } \\
\text { (years) }\end{array}$ \\
\hline $\begin{array}{c}\text { Residences } \\
\text { Comparison by site of } \\
\text { incidence for cardiac arrest }\end{array}$ & Outdoors & 11068 & 82.8 & 77.5 \\
& Schools & 98 & 10.0 & 55.7 \\
& Others & 5 & 0.7 & 49.5 \\
\hline
\end{tabular}

${ }^{\text {a}}$ Site of incidences: See Table 1. 


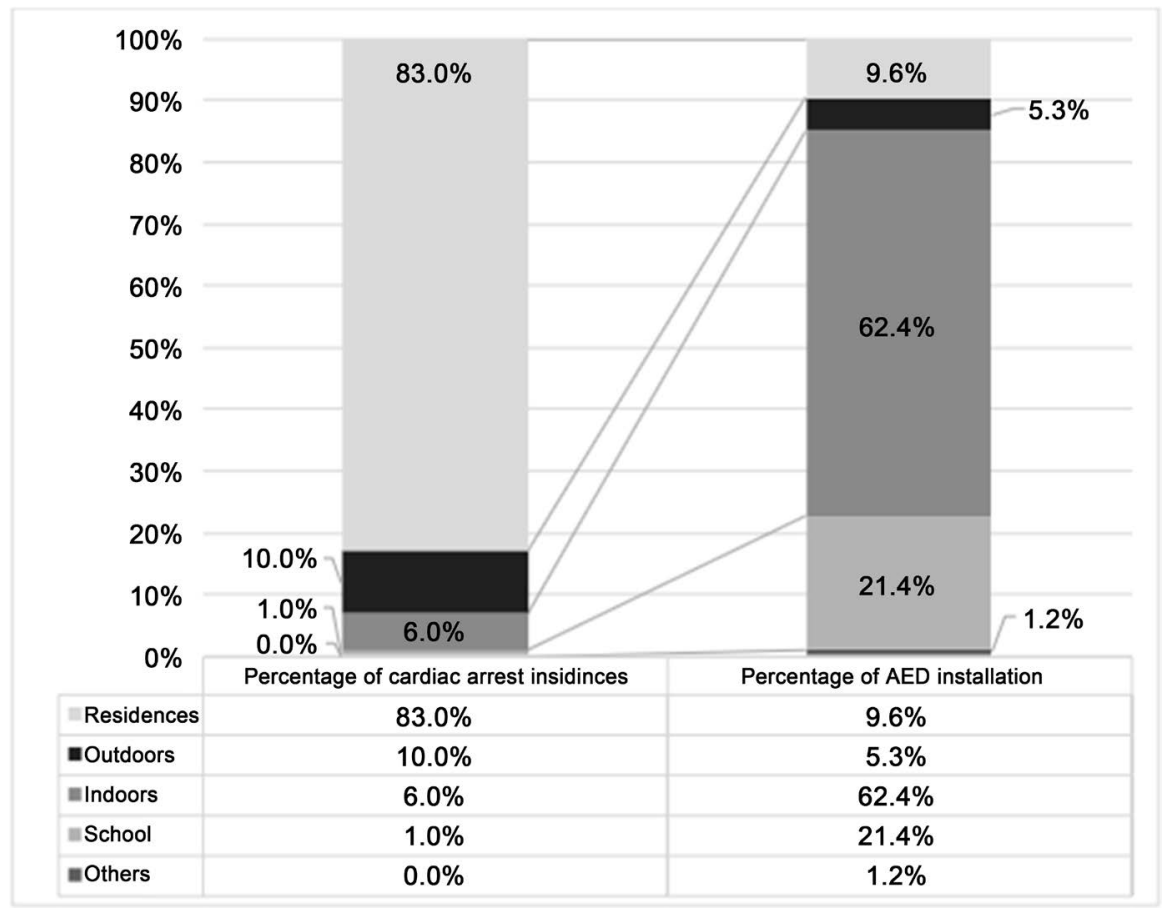

Figure 1. Comparison of percentages for sites of incidence for cardiac arrest and percentages for Location of AED installed.

\section{2) Rate of first aid and bystander's CPR}

First aid and bystander CPR rate (\%) was very high for "Schools" at 71.4\%, and followed by "Indoors" at $48.0 \%$, "Outdoors" at $28.3 \%$, "Residences" at $25.7 \%$ and "Others" at $20.0 \%$. The odds ratio of the bystander CPR rate ("Outdoors" as the standard) was highest for "Schools" at 6.35 (4.03 - 9.99).

\section{3) Rate of first aid and bystander's CPR with witnessed}

The rate of first aid and bystander's CPR with witnessed was extremely high for "Schools" at $88.6 \%$ as well, followed by "Indoors" at $72.7 \%$, "Residences" at $61.6 \%$," "Outdoors, etc." at $49.7 \%$ and "Others" at $33.3 \%$. The odds ratio bystander CPR with witnessed ("Outdoors" as the standard) was highest for "Schools" at 7.86 (3.87 - 15.96).

\section{4) Rate of defibrillation}

For the rate of defibrillation (including defibrillation by EMS) was highest for "Schools" at $46.9 \%$, "Outdoors" at $25.7 \%$, "Indoors" at $25.6 \%$ and "Others" at $20.0 \%$. "Residences" was lowest at $6.4 \%$. The odds ratio of defibrillation rate ("Outdoors" as the standard) was highest for "Schools" at 2.56 (1.69 - 3.87).

\section{5) Rate of defibrillation with witnessed}

The rate of defibrillation with witnessed was highest for schools at $58.2 \%$ as well, followed by "Outdoors" at $45.3 \%$, "Indoors" at $38.8 \%$ and "Others" at $33.3 \%$. "Residences" was the lowest at $15.4 \%$. The odds ratio of witness rate of defibrillation ("Outdoors" as the standard) was highest for "Schools" at 1.69 $(1.05-2.70)$.

6) Rate of ROCS prior to arrival to the hospital

For the rate of ROSC prior to arrival to the hospital as well, "Schools" was 
Table 3. Comparisons of OHCA occurred sites of incidence with 9 Utstein data item.

\begin{tabular}{|c|c|c|c|c|c|c|}
\hline & \multirow[b]{2}{*}{$\begin{array}{c}\text { Site of } \\
\text { incidence }\end{array}$} & \multirow[b]{2}{*}{$\begin{array}{l}\text { Total number } \\
\text { (patients) }\end{array}$} & \multicolumn{2}{|c|}{ Not witnessed } & \multicolumn{2}{|c|}{ Witnessed } \\
\hline & & & $\begin{array}{l}\text { Percentage } \\
\text { (\%) }\end{array}$ & $\begin{array}{l}\text { Odds ratio } \\
\left(95 \% \mathrm{CI}^{\mathrm{b}}\right)\end{array}$ & $\begin{array}{l}\text { Percentage } \\
\quad(\%)\end{array}$ & $\begin{array}{l}\text { Odds ratio } \\
(95 \% \mathrm{CI})\end{array}$ \\
\hline \multirow{5}{*}{ Witnessed or not } & Outdoors & 758 & 45.8 & 1.00 & - & - \\
\hline & Residences & 6414 & 41.7 & $0.54(0.48-0.61)$ & - & - \\
\hline & Indoors & 567 & 66.0 & $1.48(1.24-1.76)$ & - & - \\
\hline & Schools & 79 & 80.6 & $3.16(1.89-5.27)$ & - & - \\
\hline & Others & 3 & 60.0 & $1.14(0.19-6.84)$ & - & - \\
\hline \multirow{5}{*}{ Bystander first aid } & Outdoors & 377 & 28.3 & 1.00 & 49.7 & 1.00 \\
\hline & Residences & 2844 & 25.7 & $0.88(0.77-1.00)$ & 61.6 & $1.62(1.39-1.89)$ \\
\hline & Indoors & 421 & 48.0 & $2.34(1.96-2.80)$ & 72.7 & $2.69(2.13-3.39)$ \\
\hline & Schools & 70 & 71.4 & $6.35(4.03-9.99)$ & 88.6 & $7.86(3.87-15.96)$ \\
\hline & Others & 1 & 20.0 & $0.64(0.07-5.70)$ & 33.3 & $0.51(0.05-5.60)$ \\
\hline \multirow{5}{*}{$\begin{array}{c}\text { Defibrillation (Including } \\
\text { defibrillation by EMS) }\end{array}$} & Outdoors & 343 & 25.7 & 1.00 & 5.3 & 1.00 \\
\hline & Residences & 710 & 6.4 & $0.20(0.17-0.23)$ & 15.4 & $0.22(0.19-0.26)$ \\
\hline & Indoors & 220 & 25.6 & $1.00(0.82-1.12)$ & 38.8 & $0.77(0.61-0.96)$ \\
\hline & Schools & 46 & 46.9 & $2.56(1.69-3.87)$ & 58.2 & $1.69(1.05-2.70)$ \\
\hline & Others & 1 & 20.0 & $0.72(0.08-6.48)$ & 33.3 & $0.60(0.05-6.70)$ \\
\hline \multirow{5}{*}{$\begin{array}{l}\text { ROSC prior to arrival to } \\
\text { the hospital }\end{array}$} & Outdoors & 229 & 17.2 & 1.00 & 30.2 & 1.00 \\
\hline & Residences & 750 & 6.8 & $0.35(0.30-0.41)$ & 16.3 & $1.16(0.10-12.80)$ \\
\hline & Indoors & 206 & 24.0 & $1.52(1.23-1.88)$ & 36.3 & $1.31(1.05-1.66)$ \\
\hline & Schools & 42 & 42.9 & $3.62(2.37-5.53)$ & 53.2 & $2.62(1.64-4.19)$ \\
\hline & Others & 1 & 20.0 & $1.21(0.13-10.84)$ & 33.3 & $0.45(0.48-0.53)$ \\
\hline \multirow{5}{*}{ One-month survival rate } & Outdoors & 113 & 8.5 & 1.00 & 14.9 & 1.00 \\
\hline & Residences & 215 & 1.9 & $0.35(0.30-0.41)$ & 3.6 & $0.28(0.22-0.36)$ \\
\hline & Indoors & 91 & 10.6 & $1.28(0.96-1.71)$ & 16.0 & $1.09(0.81-1.47)$ \\
\hline & Schools & 23 & 3.5 & $3.31(2.00-5.49)$ & 29.1 & $2.34(1.39-3.96)$ \\
\hline & Others & 0 & 0.0 & - & 0.0 & - \\
\hline
\end{tabular}

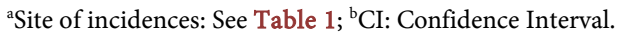

highest at $42.9 \%$, followed by "Indoors" at $24.0 \%$, "Outdoors" at $17.2 \%$, "Others" at $20.0 \%$ and "Residences" at $6.8 \%$. The odds ratio for the comparison of the rate of return of spontaneous circulation prior to hospitalization with "Outdoors" as the standard suggested that the rate of return of spontaneous circulation prior to hospitalization was highest for "Schools" at 3.62 (2.37 - 5.53).

7) Rate of ROSC prior to arrival to the hospital with witnesses

For the rate of return of spontaneous circulation prior to hospitalization when the cardiac arrest is witnessed as well, "Schools" was highest at 53.2\%, followed by "Indoors" at 36.3\%, "Outdoors" at 30.2\%, "Others" at $33.3 \%$ and "Residences" at $16.3 \%$. The odds ratio for ROSC rate prior to hospitalization with 
witnessed ("Outdoors" as the standard) was highest for "Schools" at 2.62 (1.64 4.19).

\section{8) One-month survival rate}

The one-month survival rate was highest for "Schools" at $23.5 \%$, followed by "Indoors" at $10.6 \%$, "Outdoors" at $8.5 \%$, "Residences" at $1.9 \%$ and "Others" at $0.0 \%$. The odds ratio ("Outdoors" as the standard) was highest for "Schools" at $3.31(2.00-5.49)$.

\section{9) One-month survival rate with witnesses}

For the one-month survival rate for the cardiac arrest victim when the cardiac arrest is witnessed as well, "Schools" was highest at $29.1 \%$, followed by "Indoors" at $16.0 \%$, "Outdoors" at $14.9 \%$, "Residences" at $4.6 \%$ and "Other" at $0.0 \%$. The odds ratio ("Outdoors" as the standard) was highest for "Schools" at $2.34(1.39-3.96)$.

\section{Discussion}

The OHCA occurrence sites and Installed AED location were investigated. The results showed that "Residences" were the most common site of incidence for cardiac arrest, but that AED installation for the same was lowest at 9.6\%. On the other hand, it was found that while "Schools" had the lowest incidence of cardiac arrest at 98 patients $(0.7 \%)$, the rate of the cardiac arrest with witnesses, bystander's CPR rate and one-month survival rate were conspicuously higher.

The nine Utstein item were all highest for schools and Sports institute as following which matched the report by Mitani, et al.

Witnessed cardiac arrests at schools are (97\%), with high incidents bystander's CPR (84\%), and school AEDs applied (38\%) [7]. The bystander's CPR rate , ROSC rate prior to arriving at the hospital and one-month survival rate are thought to be high at "Schools" due to the fact that a high percentage of teachers, who have a strong possibility of witnessing the cardiac arrest, have received instruction on CPR [8], and according to an investigation by the Ministry of Education, Culture, Sports, Science and Technology [9], the rate of installation of AEDs at public and private schools was $92.3 \%$ as of 2010 and is now reached to $100 \%$.

On the other hand, except for 5) rate of bystander first aid when witnessed, "Residences" had the lowest scores for six out of seven of the items among the four categories for sites of incidence, excluding "Others." Since the results were like those in the study in Osaka. [10], it was revealed that "Residences" are locations where cardiogenic cardiac arrest is likeliest to occur in Japan.

The rate of defibrillation (including that performed by ambulance service) had the lowest score for "Residences" compared to other sites of incidence. These results were like the studies by Folke, et al. [11] [12] and the study by Roger [13] that showed the application of defibrillation and chance of rescuing are higher in public spaces than residences when witnessed since the detection rate of ventricular fibrillation (VF) is higher.

Also, this study shows that installations of AEDs were most common in "In- 
doors", followed in order by "Schools" "Residences" "Outdoors" and "Others." The results showed that despite "Residences" are the most common site of incidence, the most common location of Installed AED is "Indoors". In advanced countries, the installation AEDs has been mostly recommended for public facilities [11] [12]. In Japan as well, emphasis had been placed on increasing the number of AED installations, but there has been a shift in thinking to the need to strategically deploy and manage AEDs now that the number of total installations exceeds 500,000 units. In response, the Japan Foundation for Emergency Medicine announced, "Guideline for proper placement of the AED" [14] on September 9, 2013. In addition to specific and ranked degrees of recommendations for facilities for which AED installation is recommended, the guidelines provide specific examples.

However, "Residences" where most cardiac arrests occur, are not included among the facilities recommended in the guidelines, making re-examination necessary in the future.

In the investigation by Folke, et al. [12], it was said that there should be recommendations for AEDs to be installed at $100 \mathrm{~m}$ intervals in residential areas, and in the same way, it is thought that there needs to be an examination on how to spread AEDs to "Residences" in the future in Japan as well due to the ageing of society and the increase of the population within residences.

Also, beyond direct installations in "Residences" it is thought to also be necessary to focus on taking the locations of AEDs within facilities as outlined in the "Guideline for proper placement of the AED" into consideration once more and increase awareness of AEDs that are currently installed by posting easy-to-understand signboards showing the locations of the AEDs to increase the rate of PAD.

On the other hand, increasing the number of people among the general citizens who can use AEDs will be an important factor in the effective utilization of installed AEDs. In the study by Nishiyama, et al., it was reported that the general citizens can learn CPR and how to use AEDs through even a short training [15] [16], and it will be necessary in the future to actively train people in CPR more than has been done in the past.

\section{Limitations of the Study}

This study has several limitations. This is a retrospective cohort study. It also has limitations in that public data was used in the examination to specify the sites of incidence, and no examination was made of individual cases.

\section{Conclusions}

It was revealed that mismatch between the sites of incidence for OHCA and locations of Installed AED place. Since while "Residences" were the most common sites of incidence for cardiac arrest, "Indoors" is the most common location for AED installation. On the other hand, all our data indicated that, rate of the cardiac arrest with witnesses, rate of bystander first aid, rate of defibrillation and 
one-month survival rate, were all highest for "Schools and sports institute".

These results suggest the importance of installation of AEDs that follows guidelines for the proper locating of AEDs as well as the importance of the role of school CPR/AED educational implementation lay persons.

\section{References}

[1] Director-General of the Ministry of Health, Labour and Welfare Health Policy Bureau (2004) Health Policy Bureau Notification No. 0701001: 7.1.2004.

[2] Marukawa, S., Yokota, H. and Tanabe, S. (2015) Study Report, "Study on the Spread and Education of Effective Resuscitation for Improving the Survival Rate for Cardiovascular Diseases, etc." for the 2014 Health, Labour and Welfare Scientific Study Grant (General Research Projects on Measures against Lifestyle Diseases Such as Cardiovascular Diseases and Diabetes), 2015; 40-46.

[3] Kondo, H. (2011) 2011 Study Report, "Study on the Spread and Education of Effective Resuscitation for Improving the Survival Rate for Cardiovascular Diseases, etc." for the 2011 Health, Labour and Welfare Scientific Study Grant (General Research Projects on Measures against Lifestyle Diseases Such as Cardiovascular Diseases and Diabetes), 2012; Appended Table 1.

[4] Fire and Disaster Management Agency, Ministry of Internal Affairs and Communications (2013) Current State of Emergency and Rescue Operations, 2013 Edition, 2013: Rescue Operations 80.

[5] Tokyo Fire Department: Currently Status of Emergencies (2013), 2012. 2013: 58-72

[6] Searching for Locations of Installed AED by Japan Foundation for Emergency Medicine. http://www.qqzaidan.jp/AED/aed.htm (Final Access: 6.15.2013)

[7] Mitani, Y., Ohta, K., Ichida, F., et al. (2014) Circumstances and Outcomes of Out-of-Hospital Cardiac Arrest in Elementary and Middle School Students in the Era of Public-Access Defibrillation. Circulation Journal, 78, 701-707. https://doi.org/10.1253/circj.cj-13-1162

[8] Japanese Society of School Health: 2013 Project Report on the Investigation on Health Management in School Life. 2014; Chapter 2: Investigation on Health Management in Schools, 14-18.

[9] Ministry of Education, Culture, Sports, Science and Technology: "Investigation on Measures for Safety Management at Schools" and "Installations of Automated External Defibrillators (AED) in Schools".

http://www.mext.go.jp/a_menu/gakkouanzen/syousai/1267499.htm (Final Access: 12.10.2015)

[10] Taku, I., Atsushi, H., Noriyuki, N., et al. (2006) Outcome and Characteristics of Out-of-Hospital Cardiac Arrest According to Location of Arrest: A Report from a Large-Scale, Population-Based Study in Osaka, Japan. Resuscitation, 69, 221-228. https://doi.org/10.1016/j.resuscitation.2005.08.018

[11] Folke, F., Lippert, F.K., Nielsen, S.L., et al. (2009) Location of Cardiac Arrest in a City Center: Strategic Placement of Automated External Defibrillators in Public Locations. Circulation, 120, 510-517. https://doi.org/10.1161/CIRCULATIONAHA.108.843755

[12] Folke, F., Gislason, G.H., Lippert, F.K., et al. (2010) Differences between Out-ofHospital Cardiac Arrest in Residential and Public Locations and Implications for Public-Access Defibrillation. Circulation, 122, 623-630. https://doi.org/10.1161/CIRCULATIONAHA.109.924423 
[13] Roger, A. (2010) Winkle: The Effectiveness and Cost Effectiveness of Public-Access Defibrillation. Clinical Cardiology, 33, 396-399. https://doi.org/10.1002/clc.20790

[14] Japan Foundation for Emergency Medicine: Guideline for Proper Placement of the AED. 9. 2013.

[15] Nishiyama, C., Iwami, T., Kawamura, T., et al. (2008) Effectiveness of Simplified Chest Compression-Only CPR Training for the General Public: A Randomized Controlled Trial. Resuscitation, 79, 90-96.

https://doi.org/10.1016/j.resuscitation.2008.05.009

[16] Nishiyama, C., Iwami, T., Kawamura, T., et al. (2009) Effectiveness of Simplified Chest Compression-Only CPR Training Program with or without Preparatory Self-Learning Video: A Randomized Controlled Trial. Resuscitation, 80, 1164-1168. https://doi.org/10.1016/j.resuscitation.2009.06.019

Submit or recommend next manuscript to SCIRP and we will provide best service for you:

Accepting pre-submission inquiries through Email, Facebook, LinkedIn, Twitter, etc. A wide selection of journals (inclusive of 9 subjects, more than 200 journals) Providing 24-hour high-quality service User-friendly online submission system Fair and swift peer-review system Efficient typesetting and proofreading procedure Display of the result of downloads and visits, as well as the number of cited articles Maximum dissemination of your research work

Submit your manuscript at: http://papersubmission.scirp.org/

Or contact wjcd@scirp.org 